\title{
Simulation of multigrain thin film growth
}

\author{
ANTHONY TONGEN ${ }^{\dagger}$ \\ Department of Mathematics and Statistics, James Madison University, \\ MSC 7803, Harrisonburg, VA 22807, USA \\ AND \\ DAVID L. CHOPP \\ Engineering Sciences and Applied Mathematics Department, Northwestern University \\ 2145 Sheridan Road, Evanston, IL 60208, USA
}

[Received 6 December 2004 and in revised form 24 October 2005]

\begin{abstract}
In [28], Russo and Smereka presented a level set method for the growth of fully faceted grains. This method produced interesting results, but it is not applicable to typical grain growth where the operating temperature is well below that which would produce fully faceted grains during deposition. In this lower temperature regime, the deposition rate and the diffusion rate are in competition to determine the ultimate texture of the film, and which grain orientation is preferred. In this paper, we present a new technique coupling the level set method with the marker particle method to study multigrain thin film growth. The resulting method is designed for more realistic temperature ranges where grains are not typically fully faceted.
\end{abstract}

\section{Introduction}

Numerical simulations involving grain growth are extremely important yet very difficult to model due to the numerous factors contributing to the growth process. Most of the previous work has focused on simulations in unique temperature regimes. In fact, Russo and Smereka [28] developed a level set formalism for the motion of faceted interfaces. Their main assumption was that grain growth only depends on the orientation of the grain facet; this assumption implies that temperatures are well above typical operating conditions. Bloomfield et al. [7] developed a three-dimensional extension of the Russo-Smereka model [28]. However, both of these simulations neglect three very important aspects of grain growth: spatially-dependent deposition, contact angle between grains, and temperature.

This paper focuses on adding to the basic framework of evolution of grain growth using the level set method while implementing more physically relevant parameters. We are able to capture the very important feature of texture competition in multigrain thin film growth using the numerical simulation designed in this paper. Furthermore, we have duplicated the experimentally observed result that higher temperature deposition leads to dominance of $\langle 100\rangle$ grain orientation, while lower temperatures lead to $\langle 111\rangle$ orientation dominance (see e.g. [19, 27]).

The tendency of grains to develop a preferred orientation is a significant aspect of thin film growth. There is often a strong correlation between texture and performance. For instance, $\langle 111\rangle$ is

\footnotetext{
${ }^{\dagger}$ This work was supported in part by NSF-IGERT DGE-98-70659. E-mail: tongen@math.jmu.edu

This work was supported in part by NSF DMS-96-15877. E-mail: chopp@ northwestern.edu
} 
desirable in metallic interconnects of semiconductors and $\langle 100\rangle$ is preferred for diffusion barriers and other mechanical applications [19]. Modern integrated circuits whose interconnects are made of copper and aluminum thin films exemplify the importance of texture in film growth [41]. Experiments have shown that the durability of an interconnect is a function of its microstructure. It has also been shown that the $\langle 111\rangle$ texture is most effective in suppressing electromigration. The present work examines the behavior of thin film growth.

The level set method has frequently been used in materials modeling due to its applicability in areas such as deposition and etching [1, 6, 20]. However, one reason the level set method is popular in materials science computation is the fact that topological changes are handled naturally. Therefore, the level set method is a logical choice for handling deposition of multiple grains. Other methods must elaborately remesh in order to manage the joining of multiple grains, while the level set method implementation is straightforward.

In addition, the level set method is utilized because we are able to overcome one of the weaknesses of continuum modeling. As will be described later, each grain is represented by a different level set function. Therefore, we observe not only the evolution of the entire interface, but also the interface of each individual grain. Thus, we are better able to identify the evolution of grain boundaries.

\section{The physical problem}

This study uses a continuum model to study the three major driving forces for the surface morphology of thin film growth: surface diffusion, deposition and contact angle. Due to the importance of preferred orientations during film growth, we will focus on the two major orientations that dominate, namely $\langle 111\rangle$ and $\langle 100\rangle$. Figure 1 displays the alignment of the $\langle 111\rangle$ and $\langle 100\rangle$ orientations. To more easily differentiate the competition between the two orientations, we will rotate the $\langle 111\rangle$ orientation $45^{\circ}$ in two coordinate directions so that it is perpendicular to the $\langle 100\rangle$ orientation.

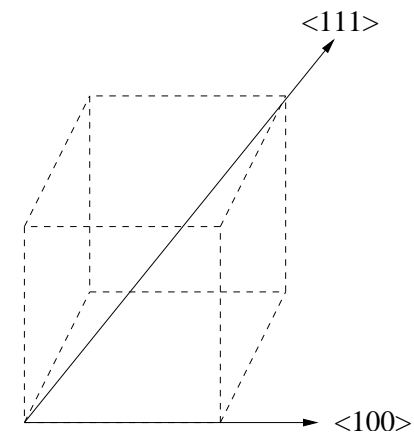

FIG. 1. Actual orientations of the $\langle 111\rangle$ and $\langle 100\rangle$ textures.

The setting for this model is a box $\Omega \subset \mathbb{R}^{2}$, in which two grains, $\Omega_{1}(t)$ and $\Omega_{2}(t)$, evolve. For the present study, we will assume that $\Omega_{i}(t)$ represent grains with different preferred orientations. The boundary of $\Omega_{i}(t)$ consists of two parts: one part which is in touch with the adjacent grain whose normal velocity is zero and another part which evolves according to the aforementioned primary driving forces. 


\subsection{Deposition}

This paper closely follows the work done in [1, 2, 3] to model deposition. As shown in Figure 2, it is assumed that deposition is from a line source, which can represent both sputter deposition and angle-based flux functions. Consequently, the general equation for deposition normal velocity is given by

$$
F_{\text {deposition }}=\iint_{\text {visible region }} \frac{f(\theta) \cos (\xi)}{r^{2}} \mathrm{~d} A,
$$

where $r$ is the distance between the discrete surface element and the discrete line source element, $\theta$ is the angle between the normal to the discrete surface element and the line of length $r$, and $\xi$ is the angle between the normal to the discrete line source element and the line of length $r$. The term $\cos (\xi)$ implies that the substrate directly below the target receives the most deposition, but the nearby substrate still receives material. Also, the substrate that is further from the target receives approximately $1 / r^{2}$ less material, which decreases rapidly as $r$ increases. Line source deposition can be generalized to model many types of deposition, such as molecular beam epitaxy, physical vapor deposition, and other deposition techniques.

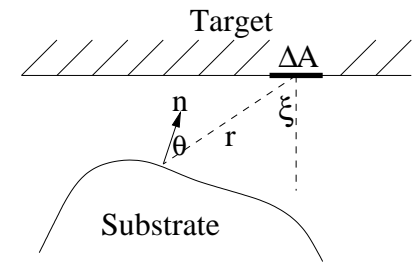

FIG. 2. Pictorial representation of the method of deposition.

The value of $f(\theta)$ is determined by the deposition technique being modeled. For instance, isotropic deposition is modeled with $f(\theta)=1$; this assumption emphasizes the importance of normal deposition from the target. We will model deposition with $f(\theta)=\cos (\theta)$. This model has a similar interpretation to the choice of $\cos (\xi)$, except that there is a balance between the normal deposition of the target and normal reception of the substrate.

\subsection{Surface diffusion}

Surface diffusion is an important component in many mathematical models of materials, such as metal reflow in semiconductor manufacturing [26], sintering [40], thin-film deposition [17], and elastic membrane simulations [18]. While it may or may not be the primary driving force in a numerical simulation of these processes, surface diffusion is very often the rate limiting step when it is simulated with a numerical method. Isotropic surface diffusion is often modeled by the fourthorder nonlinear differential operator

$$
\dot{\mathbf{x}}=-\left(\nabla_{s} \cdot\left(\nabla_{s} \kappa\right)\right) \mathbf{n}(\mathbf{x}),
$$

where $\mathbf{x}$ is a point on the material surface, $\mathbf{n}(\mathbf{x})$ is the outward normal to the surface at $\mathbf{x}, \kappa$ is the mean curvature of the surface, and $\nabla_{s}$ is the surface gradient [23].

There are two different types of diffusion that play critical roles in coverage and deposition processes: bulk diffusion, which is related to the global motion of the material throughout the 
deposited layer; and surface diffusion, which is related to the motion of the surface boundary. It is well known that surface diffusion effects are some of the most important features of ultra thin film equilibrium shapes [42]. The influence of surface diffusion can be seen during both crystal faceting and grain boundary grooving. For anisotropic crystals, the existence of growth competition can be directly related to surface diffusion.

Cahn and Taylor [8] have generalized the earlier work of Mullins [23] by defining new laws that constitute a class of dynamic problems where: volume is conserved; the only energy in the system is surface energy; and the only driving force for surface motion is surface energy reduction. Their model consists of a fourth order nonlinear partial differential equation involving the surface Laplacian of the mean curvature. Numerical solutions are difficult to compute due to the fourthorder derivatives in (2). Using an explicit method to solve Laplacian of curvature flow implies that the time step scales with the space step to the fourth power, i.e. $\Delta t \sim \Delta x^{4}$. Numerical stability for such an equation requires very small time steps, making the simulation of the surface diffusion process computationally expensive. The other option is to solve (2) using an implicit method, but implementation is difficult due to the nonlinearity.

Consider a given material undergoing surface diffusion at temperature $T$, with diffusivity $D(\theta, T)$ (a function of the angle of the surface normal $\theta$ and temperature $T$ ), surface energy $\gamma(\theta)$, and mean curvature $\kappa$. The general surface diffusion normal velocity is given by

$$
F_{s d}=-\nabla_{s} \cdot\left[D(\theta, T) \nabla_{s}\left(\left(\gamma(\theta)+\gamma^{\prime \prime}(\theta)\right) \kappa\right)\right] .
$$

Isotropic surface diffusion, as given by (2), is a special case of (3), with $D(\theta, T)=\gamma(\theta) \equiv 1$. If $D(\theta, T)$ or $\gamma(\theta)$ vary with respect to $\theta$, the process is called anisotropic.

Surface diffusion is related to the motion of the boundaries of a thin film. The diffusivity is usually written in the form

$$
D(\theta, T)=D_{0}(\theta) \exp \left(\frac{-E_{m}}{k_{B} T}\right)
$$

where $D_{0}$ is the diffusion constant, $E_{m}$ is the activation energy, $k_{B}$ is Boltzmann's constant, and $T$ is the temperature. Both $D_{0}$ and $E_{m}$ are estimated with values that depend on the material being deposited.

Previous work has been done using the level set method to model thin film growth. As previously mentioned, Russo and Smereka [28] computed the motion of multiple grains for the limiting hightemperature case where all grains remain fully faceted. Also, in [1, 2, 3] they present device scale simulations in two and three dimensions that model both the etching and deposition processes. More recently, the level set method was used to study physical vapor deposition and device processing. The present study builds upon the aforementioned work to develop a model for grain growth and to reproduce the texture competition that has been verified experimentally [19].

\section{Numerical methods}

In the present simulations we use a combination of the marker particle method and the level set method. Here we present a description of both methods.

\subsection{Marker particle method}

The marker particle method is a straightforward method for the solution of interface propagation problems. Equally spaced marker points are placed on the discretized interface. Then using the laws 
of motion, each marker point is evolved according to the prescribed speed function. Given a speed function $F$ normal to the curve, we can write an evolution equation for any point on the curve $\Gamma$ as

$$
\frac{\mathrm{d}}{\mathrm{d} t} \mathbf{r}=F \cdot \mathbf{n}=F \frac{\left(y_{s},-x_{s}\right)}{\left(x_{s}^{2}+y_{s}^{2}\right)^{1 / 2}},
$$

where $\mathbf{r}(s, t)=(x(s, t), y(s, t))$. Converting this equation into its individual components, we arrive at the representation for the motion of a point $(x(s, t), y(s, t))$ with normal velocity $F$ given by

$$
x_{t}(s, t)=\frac{F y_{s}}{\left(x_{s}^{2}+y_{s}^{2}\right)^{1 / 2}}, \quad y_{t}(s, t)=\frac{-F x_{s}}{\left(x_{s}^{2}+y_{s}^{2}\right)^{1 / 2}} .
$$

The equations of motion for the marker particle method are a coupled system of partial differential equations. After the marker points have been moved using (5), the interface is reconstructed by reconnecting the individual points with either straight lines or splines. A similar procedure could be used in three dimensions except triangles would be used to reconstruct the surface.

The marker particle method has several advantages. First, it is straightforward to implement for any given speed function. Second, it is fast, since (5) is only evolved at the discretized points on the curve. Third, higher order accuracy is easily obtained through standard numerical methods for ordinary differential equations.

On the other hand, stability can be difficult to maintain due to irregular spacing of the nodal points. In order for interfaces to merge in the marker particle method, an elaborate method of collision detection along with addition and subtraction of nodal points must take place. As a result, complicated re-meshing strategies are needed near points where topological changes occur. This problem becomes even more pronounced in three dimensions.

\subsection{The level set method}

One of the most versatile and effective ways of computing the motion of curves and surfaces is the level set method developed by Osher and Sethian [25]. The calculations and numerical algorithms introduced in [25, 11, 30, 31] have provided the basis for a large collection of calculations in such areas as combustion and fluid mechanics [9, 37, 43], medical imaging [22], crack propagation [34, 36, 33], materials science [20, 12, 13], and etching and deposition in semi-conductor manufacturing [1, 2, 3]. For a review and resources on level set methods, see [24, 29].

In the level set method, the interface of interest is embedded in a higher dimensional function. The main advantages of the level set method are that topological changes are handled naturally, the technique easily extends to three and higher dimensions, and finite difference schemes can be used for approximation operators on a fixed Eulerian mesh.

3.2.1 The general level set formulation. Imagine a closed curve $\Gamma(t)$ in the plane propagating normal to itself with speed $F$. We can embed the initial position, $\Gamma_{0}$, of $\Gamma(t)$ in terms of a higher dimensional function $\phi$, i.e. $\Gamma_{0}=\left\{x \mid \phi_{0}(x)=0\right\}$. The evolution of $\Gamma$ and the evolution of $\phi$ are connected through the equation

$$
\phi_{t}+F|\nabla \phi|=0, \quad \phi(x, 0)=\phi_{0}(x),
$$

as derived in [25]. At any time, the front $\Gamma$ is given by the zero level set of the time-dependent level set function $\phi$. 
The initial conditions on $\phi$ are usually given by the signed distance function:

$$
\phi_{0}(x)= \pm \min _{y \in \Gamma_{0}}|x-y|,
$$

where the sign of $\phi$ depends on whether $x$ is inside $(\phi<0)$ or outside $(\phi>0)$ of $\Gamma$. Figure 3 is an example of a signed distance function when $\Gamma$ is a circle. The solution of 6 is best accomplished using schemes borrowed from methods for solving hyperbolic conservation laws.

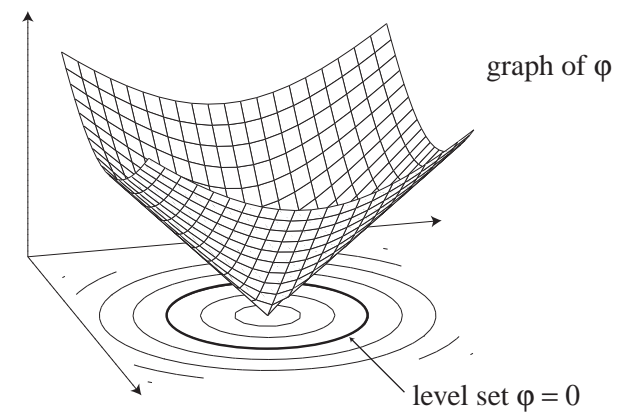

FIG. 3. Level set representation of the signed distance function for a circle.

Formulae for geometric quantities such as the normal, curvature, and Laplacian of curvature of the curve $\Gamma$ can all be derived in terms of the level set function $\phi$, as shown below for a onedimensional curve in the plane:

$$
\begin{aligned}
\mathbf{n} & =\frac{\nabla \phi}{|\nabla \phi|}=\frac{\left(\phi_{x}, \phi_{y}\right)}{\sqrt{\phi_{x}^{2}+\phi_{y}^{2}}}, \\
\kappa & =\nabla_{s} \cdot \nabla_{s} \mathbf{n}=\frac{\phi_{x x} \phi_{y}^{2}-2 \phi_{x y} \phi_{x} \phi_{y}+\phi_{y y} \phi_{x}^{2}}{\left(\phi_{x}^{2}+\phi_{y}^{2}\right)^{3 / 2}}, \\
\kappa_{s s} & =\nabla_{s} \cdot \nabla_{s} \kappa=\frac{\kappa_{x x} \phi_{y}^{2}-2 \kappa_{x y} \phi_{x} \phi_{y}+\kappa_{y y} \phi_{x}^{2}}{\phi_{x}^{2}+\phi_{y}^{2}}-\frac{\left(\kappa_{x} \phi_{x}+\kappa_{y} \phi_{y}\right) \kappa}{\left(\phi_{x}^{2}+\phi_{y}^{2}\right)^{1 / 2}},
\end{aligned}
$$

where $\nabla_{s}$ is the surface gradient [14]. All of these equations can be discretized using standard finite difference schemes.

In equation (6), $F$ is often given as a function of the normal velocity on the surface $\Gamma(t)$. However, (6) requires knowledge of $F$ in the entire domain of $\phi$. This problem will be handled by using velocity extensions.

3.2.2 Velocity extensions and the fast marching method. Note that (1) only defines the speed function $F$ on the interface, $\phi^{-1}(0)$, while $(6)$ requires $F$ to be defined on the whole domain. Consequently, we define $F_{\text {ext }}$ to be the speed function used in (6),

$$
\phi_{t}+F_{\text {ext }}|\nabla \phi|=0
$$

with the additional condition that

$$
\left.F_{\text {ext }}\right|_{\phi^{-1}(0)}=F
$$

This new velocity field $F_{\text {ext }}$ is known as the extension velocity. 
Of course, there are many choices for $F_{\text {ext }}$. However, for stability purposes, it is advantageous to choose $F_{\text {ext }}$ in such a way that $\phi$ is maintained as a signed distance function. It was shown in [4] that this results in the additional condition

$$
\nabla F_{\mathrm{ext}} \cdot \nabla \phi=0
$$

A geometric interpretation of $[13$ is that the velocity field is constant along lines normal to the interface, $\phi^{-1}(0)$.

In [4], a technique was introduced for building this extension velocity field, $F_{\text {ext }}$, which satisfies the conditions (12), (13), from a velocity field $F$ given on the front $\phi=0$. This technique relied on the fast marching method [32], which is an optimal technique for solving the eikonal equation.

Briefly, the fast marching method solves an equation of the form

$$
G(x, y)|\nabla \phi|=1
$$

by first replacing the gradient with suitable upwind operators, and then systematically advancing the front by marching outwards from the boundary data in an upwind fashion. The key to the algorithm lies in the observation that an upwind operator implies a causality; hence grid points with a given value for $u$ cannot be affected by grid points with a bigger value for $u$. Therefore, as the solution is advanced, a heap sort is used to monitor the grid point with the smallest $u$ value to be updated, and thus always advances the solution "downwind" of that point. Through the use of this sorting algorithm, each point in the domain is visited only once, rather than requiring more iterations. The resulting technique has a total operation count of $O(N \log N)$.

In our algorithm, the fast marching method is used to solve the equation for the signed distance,

$$
|\nabla \phi|=1
$$

by initializing grid points near the interface using direct computation of the signed distance to the interface, then using the fast marching method to compute $\phi$ at the remaining grid points. At the same time, the extension velocity, $F_{\text {ext }}$, is initialized by tracing orthogonally from the grid points near the interface back to the interface $\phi^{-1}(0)$ where the original speed function, $F$, is determined. The remainder of the values of $F_{\text {ext }}$ in the domain are computed in the same order as those for the $\phi$, by discretizing $(13)$ in the same upwind directions determined by the fast marching method. Each time the deposition velocity, (1), is computed this velocity extension technique is used to create $F_{\text {ext }}$ to advance $\phi$ according to $(11)$.

For a complete description of the level set method, the fast marching method, velocity extensions, and some of their applications, see [24, 29].

\subsection{Surface diffusion implementation}

The annealing process in grain structure is very critical in micro-electronic devices. Most valuable electrical properties of micro-electronic devices are related to the interface morphology or roughness. It has been shown experimentally that the annealing process smooths material surfaces. We will study the effect of annealing on the surface roughness of the grains. Atomic scale simulations of annealing can be found in [38, 39].

For simulating two-dimensional surface diffusion, we will use the definition by Cahn-Taylor [8]. Consider a given material undergoing surface diffusion at temperature $T$, with diffusivity $D(\theta, T)$, where $\theta$ is the angle of the surface normal, $\gamma(\theta)$ the surface energy, and $\kappa$ the mean curvature. The 
surface diffusion normal velocity is given by

$$
F_{s d}=-\nabla_{s} \cdot\left[D(\theta, T) \nabla_{s}\left(\left(\gamma(\theta)+\gamma^{\prime \prime}(\theta)\right) \kappa\right)\right] .
$$

Although the model is material independent, we will use parameters measured for deposition of aluminum on silicon. Using material parameters from [5, 15, 35] for aluminum, we approximate the diffusivity as

$$
D(\theta, T)=D_{0}(\theta) e^{-\frac{0.985-.035 \cos (2 \theta)}{k_{B} \cdot T}} \mathrm{~nm}^{2} / \mathrm{s},
$$

where $D_{0}(\theta)=(2.52+2.48 \cos (2 \theta)) \cdot 10^{12}, k_{B}$ is the Boltzmann constant, and $T$ is the temperature. We approximate the surface free energy as

$$
\begin{aligned}
& \gamma(\theta)_{\langle 100\rangle}=0.075-0.005 \cos (4 \theta), \\
& \gamma(\theta)_{\langle 111\rangle}=0.075-0.005 \cos (4 \theta+\pi),
\end{aligned}
$$

where surface energy is defined for the two orientations under examination.

We will use a marker particle method to simulate surface diffusion for its computational speed [14]. For comparison, if we compute surface diffusion using the level set method on a $100 \times 100$ grid, (16) must be calculated at 10,000 grid points. However, a comparable marker particle representation will have approximately 200-300 marker particle points where (16) must be calculated. The marker particle method is used because the surface diffusion step is the biggest computational bottleneck in the simulation.

The marker particle simulation of surface diffusion adequately accounts for the critical points of the surface free energy in both (18) and (19). The critical points occur when $\gamma+\gamma_{\theta \theta}=0$. In order to obtain correct evolution of the surface, we placed marker points on the curve where the critical angle passes across a facet. This adaptation, coupled with the fact that the interface does not evolve long enough to become unstable, is sufficient to correctly compute surface diffusion even at the critical points of the surface free energy.

\subsection{Contact angle}

It is well known that a contact angle exists in thin film deposition [21, 16]. Material deposits on the substrate in such a way that a contact angle is present between the two grains. Physically, the contact angle controls the separation between grains, thereby influencing the amount of deposition near the point of intersection of the grains. For instance, a small contact angle results in a small separation between grains, causing more shadowing near the point of intersection and less deposition.

For a well-posed model involving surface diffusion with boundary conditions, two boundary conditions must be imposed at each end point. In the present situation, the end point is defined as the location of the point of contact between the evolving surface and the underlying solid. The boundary conditions are a no-flux condition and a prescribed contact angle between the surface and the fixed solid. The no-flux condition is enforced by prescribing

$$
\nabla\left[\left(\gamma+\gamma_{\theta \theta}\right) \kappa\right]=0
$$

at the endpoints. Equation (20) is a Neumann type boundary condition which prevents flux due to surface diffusion through the grain boundary (see (3)). The contact angle is enforced numerically by 
1. Finding the location of the points of contact.

2. Evolving all points on the interface, except the points of contact, by 16$)$ with the no-flux condition.

3. Moving the points of contact so that the prescribed contact angle is maintained.

This algorithm is enforced for each grain. Alternating between the grains ensures that the motion between the grains is based on surface diffusion rather than a numerical bias.

Mass conservation is a concern during this aspect of the computation; however, mass conservation is more accurate as the resolution increases. Also, experimental verification is the driving force for the contact angle and any mass that we are gaining or losing is due to this experimental reproduction. We consider any mass gain/loss at this step to be a part of the model rather than an artifact of the computation. Overall, the contact angle mass preservation is approximately first order, which agrees with the rest of the computational model.

\subsection{Deposition implementation}

We follow closely the work done in [1, 2, 3] to model the process of deposition. In our problem, we assume deposition is from a line source. This model can be used for both sputter deposition and angle-based flux functions. Recall that the equation for deposition normal to the interface is

$$
F_{\text {deposition }}=\iint_{\text {visible region }} \frac{f(\theta) \cos (\xi)}{r^{2}} \mathrm{~d} A,
$$

where: $r$ is the distance between the discrete surface element and the discrete line source element; $\theta$ is the angle between the normal to the discrete surface element and the line of length $r$; and $\xi$ is the angle between the normal to the discrete line source element and the line of length $r$. Figure 4 is a pictorial representation of the model for deposition.

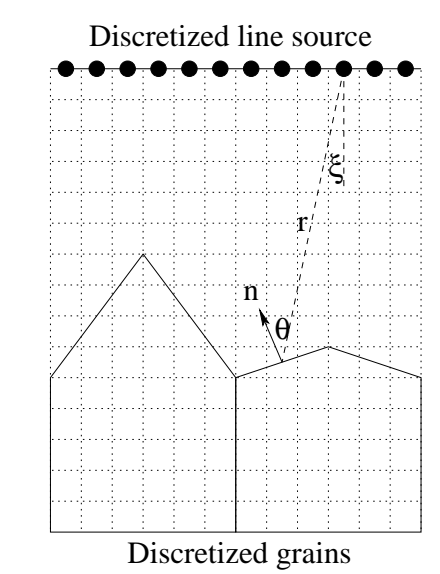

FIG. 4. Representation for our complete model.

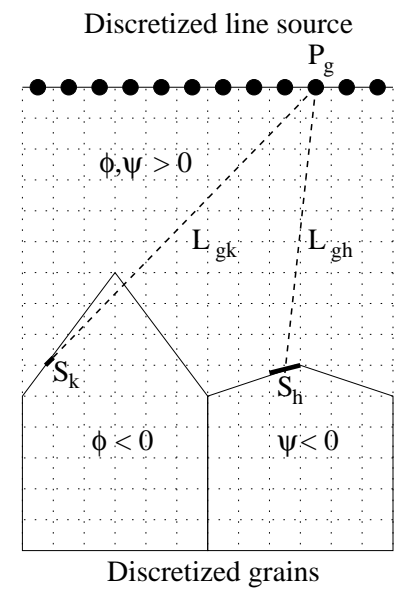

FIG. 5. Depiction of visibility calculation. $S_{h}$ is visible, since $\phi, \psi>0$ for all of $L_{g h} . S_{k}$ is not visible, since $\phi<0$ where $L_{g k}$ passes through the left grain.

We will be simulating deposition with the level set method. This implementation is advantageous because the value of the level set function at each point in the domain determines whether a point is 
inside or outside of the grain. In Figure 5, we denote $\phi, \psi<0$ as inside of the grains and $\phi, \psi>0$ as outside of the grains. The following is an algorithm for visibility in general and for the two cases shown in Figure 5

1. Discretize the line source into $i$ different point sources, $P_{i}$. Consider the point source $P_{g}$.

2. Discretize both grains into points such that a point is found where the interface crosses the mesh. Make $j$ segments, $S_{j}$, such that a segment consists of neighboring points. Consider two segments $S_{h}$ and $S_{k}$.

3. Make a line, $L_{i j}$, from $P_{i}$ to the midpoint of $S_{j}$. Consider the two lines $L_{g h}$ and $L_{g k}$.

4. Discretize $L_{i j}$ into $N$ points. At each discretized point, check the sign of $\phi$ and $\psi$.

5. If $\phi, \psi \geqslant 0$ for all points on the discretized line, then $S_{j}$ is visible from $P_{i}$. Notice that all points on $L_{g h}$ have $\phi, \psi \geqslant 0$, so $S_{h}$ is visible from $P_{g}$.

If $\phi<0$ or $\psi<0$ at a single point on the discretized line, then $S_{j}$ is not visible from $P_{i}$. Notice that $L_{g k}$ has a portion where $\phi<0$, so $S_{k}$ is not visible from $P_{g}$.

6. Repeat steps 3-5 for all points on $P_{i}$ and $S_{j}$.

This algorithm requires $N K L$ computations per deposition step, where $K$ is the number of edge segments and $L$ is the number of source segments. Therefore, the deposition process is also computationally expensive. However, using the level set method increases computational efficiency. Rather than discretizing the line and checking many unnecessary points, recall that the level set function at a given point provides the distance to the interface. This feature of the level set method allows much larger steps in the visibility calculation for deposition. In the following computations, a combination of both methods will be implemented. We take one fairly large step based on the level set value and then use the aforementioned algorithm as we approach the interface.

\subsection{Algorithm}

In this paper, we use the marker particle method to handle the surface diffusion process in which little, if any, merging occurs. We use the level set method to handle the deposition process in which merging and visibility calculations occur.

One concern of using two different representations of the interface is artificial diffusion, which is caused by using the level set method to find the front. This concern is addressed by using a higher order method for subgrid resolution of the level set function found in [10].

The marker particle time step restriction for surface diffusion is $O\left(\Delta t / \Delta x^{4}\right)$ because of the fourth-order derivative, but the deposition time step can be much larger. Due to this difference in time steps, we use a split step approach in which we evolve the grain in the marker particle scheme for many steps between each deposition step, thus reducing the number of times that we need to alternate between the two methods. Recall that the computational cost for visibility is quite substantial. However, since the time step restriction for deposition can be much larger, there is an opportunity to balance the computational cost with the size of the time step. This procedure helps to minimize the artificial diffusion caused by using two different methods.

Our algorithm for the deposition and annealing problem is as follows:

1. Initialize the level set function for each grain, $\Omega_{i}(t)$.

2. Advance by deposition (1) in the level set method with time step $\Delta t, \Omega_{i+1}(t)=\Omega_{i}(t)+$ $\Delta t F_{\text {deposition }}$. 
3. Create a marker particle representation of the zero contour of the level set function and advance by anisotropic surface diffusion (3) using $N$ steps with the time step $\Delta t / N, \Omega_{i+1}(t)=\Omega_{i}(t)+$ $\Delta t F_{s d}$.

4. Convert the marker particle representation back into a level set function.

5. Go to step 2.

When solving the level set equation, we are using a first-order implementation. Likewise, we are using a first-order method for the marker particle method. Overall, the numerical scheme, including the computation of the contact angle, is first-order accurate.

While we are able to demonstrate first-order convergence, one potential issue regarding the accuracy of this method concerns the relative accuracy of the bicubic interpolation compared to the motion due to surface diffusion. To verify that the results we obtain are due to surface diffusion, and not numerical diffusion from reinitialization, we performed a numerical experiment. Begin with a circle and evolve the surface by straight surface diffusion and by surface diffusion with periodic transfers to a level set mesh, and then back to the marker particle representation. The number of transfers back and forth is chosen to be proportional to what is done in the full algorithm. Figure 6 shows the results of this calculation and how the periodic use of reinitializations does not significantly impact the motion. It is worth noting that these results can degrade some in the case where orders of magnitude more reinitializations are used. In terms of our results, this would correspond to very low temperature cases, where the diffusion rates are significantly smaller than those that we explored.

We should also note that this algorithm is easily extended to fully three-dimensional computations. The primary difficulties in extending this method to higher dimensions are possible topology changes occurring in the marker particle framework, and the cost of the deposition calculation. The first difficulty is not of concern because the topology changes are handled by the level set method through the operator splitting. The second difficulty is strictly computational cost and not an algorithmic challenge.

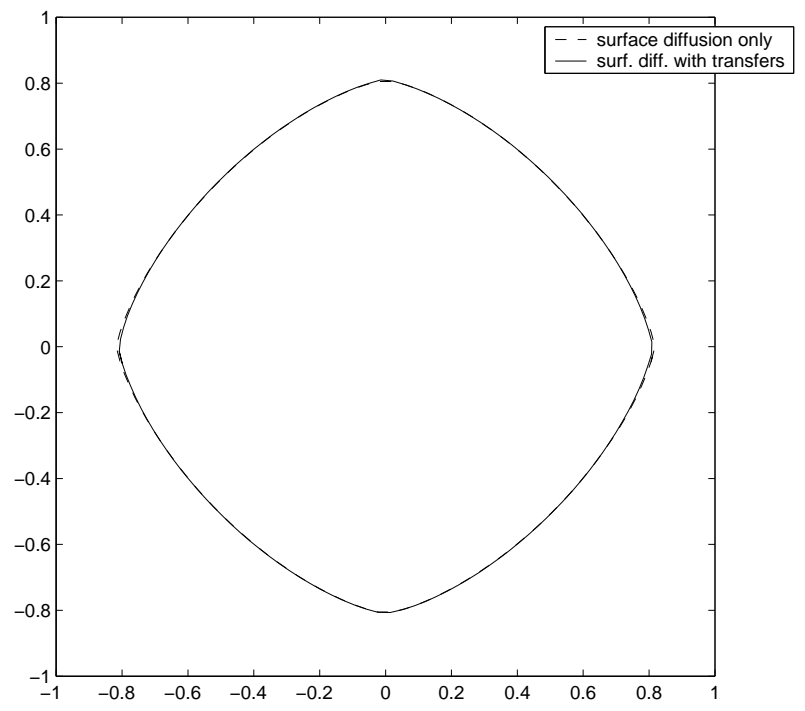

FIG. 6. Comparison between simple surface diffusion and surface diffusion with periodic reinitializations. 


\section{Results}

In the discussion that follows, we present some results about single and multiple grain growth under the effects of surface diffusion, the deposition process, temperature and contact angle. For the rest of this section we will refer to angles according to Figure 7 . We denote by $\alpha_{\langle 111\rangle}$ and $\alpha_{\langle 100\rangle}$ the angles at the top of the $\langle 111\rangle$ and $\langle 100\rangle$ grains, respectively, and by $\theta_{c}$ the contact angle.

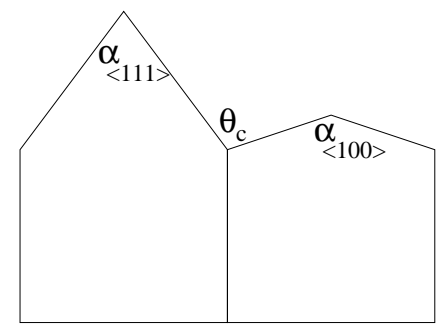

FIG. 7. Depiction of angle terminology.

The case of contiguous grains with different anisotropy has a critical importance in grain growth modeling. A realistic two-dimensional representation is a combination of contiguous grains with different orientations. Considering two grains with different orientations, we can accurately check the validity of our periodic boundary approach. We make the simulation periodic in the direction parallel to the width of the grain. This periodicity allows the line source to deposit through the boundary of the domain, producing an infinite line source with an infinite number of grains.

To be useful for grain and device simulations, our model must give valuable results consistent with experimental data from the annealing and deposition processes. The crux of the modeling is to look at the influence that temperature, contact angle, deposition and surface diffusion have on grain growth. The two most important cases are high temperature, which occurs at approximately $T=700 \mathrm{~K}$, and low temperature, which occurs at approximately room temperature or $T=300 \mathrm{~K}$. All of the following simulations have a computational domain that is approximately $4 \mathrm{~nm}$ in width and $3 \mathrm{~nm}$ in height.

Consider the effect that temperature has on diffusivity. Recall that we modeled diffusivity as

$$
D(\theta)=D_{0}(\theta) e^{-\frac{0.985-.035 \cos (2 \theta)}{k_{B} \cdot T}} \mathrm{~nm}^{2} / \mathrm{s}
$$

where $D_{0}=(2.52+2.48 \cos (2 \theta)) \cdot 10^{12}$.

The curves for diffusivity as a function of the angle $\theta$ have the same shape, but an important difference between the high temperature case and low temperature case is the value of the diffusivity. When $T=700 \mathrm{~K}$, diffusivity is on the order of $10^{5} \mathrm{~nm}^{2} / \mathrm{s}$ whereas when $T=300 \mathrm{~K}$, diffusivity is on the order of $10^{-4} \mathrm{~nm}^{2} / \mathrm{s}$. Since the deposition rate is relatively similar in both situations, one can clearly see that when $T=700 \mathrm{~K}$, diffusion dominates, while at $T=300 \mathrm{~K}$ deposition dominates.

Now that we have identified the key distinction between high and low temperature experiments, we will examine the numerical simulations. We will also examine the viability of our model to reproduce the desired texture competition.

We will consider two different initial conditions: the first will be equal initial conditions, i.e. $\alpha_{\langle 111\rangle}=\alpha_{\langle 100\rangle}=135^{\circ}$, and the second will represent the more physically relevant alignment by 
initializing the orientations to be small perturbations of their desired orientations. A series of results representing the aforementioned cases can be seen in Figures 8 and 9 , respectively. We will begin our analysis by examining the extremal temperature cases while varying the contact angle.

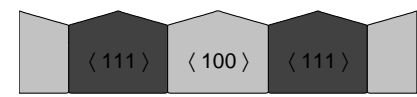

(c)

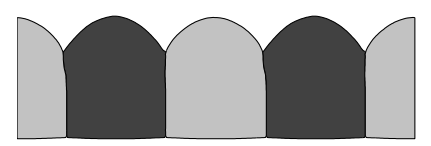

(e)

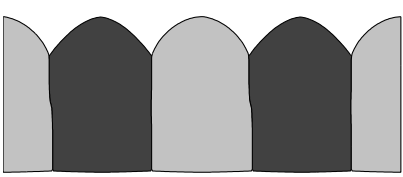

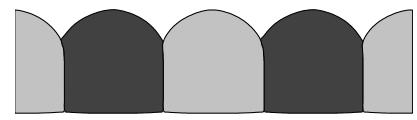

(d)
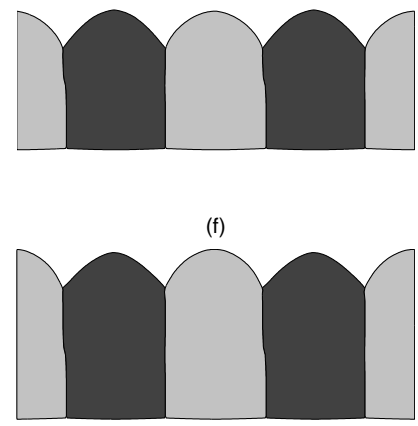

FIG. 8. Evolution of grain growth for $\theta_{c}=2 \pi / 3$ and $T=300 \mathrm{~K}$ with equal initial conditions for each grain.

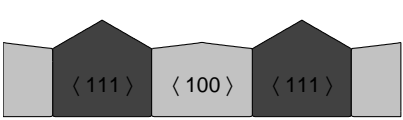

(c)

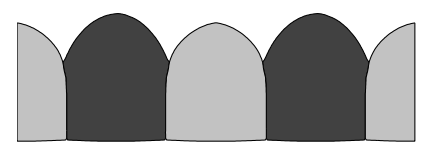

(e)

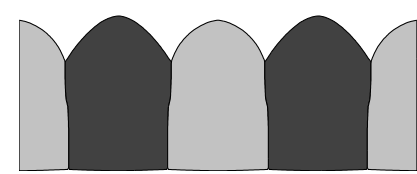

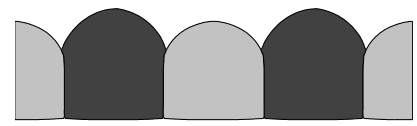

(d)

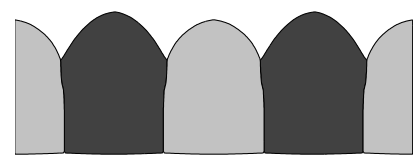

(f)

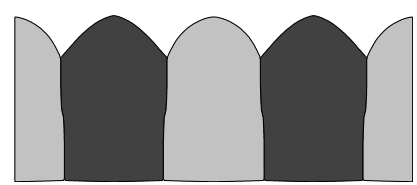

FIG. 9. Evolution of grain growth for $\theta_{c}=2 \pi / 3$ and $T=300 \mathrm{~K}$ with initial conditions that are small perturbations of the desired orientations for each grain. 
We first examine $T=300 \mathrm{~K}$ where deposition dominates. Recall that at low temperatures, the $\langle 111\rangle$ orientation should dominate, and the resulting growth should be columnar. Dominance of the $\langle 111\rangle$ orientation is demonstrated by greater height and area than the $\langle 100\rangle$ orientation.

Examining the results from Figures 10 and 11 , we see very similar behavior between the two figures. Regardless of the initial configuration of the grains, the results with $\theta_{c}=120^{\circ}$ reproduce the expected experimental results. The larger contact angle allows for more deposition at the grain boundaries which produces $\langle 111\rangle$ dominance.

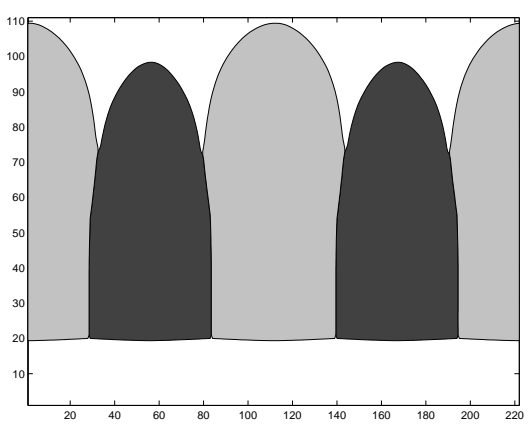

(a) $\theta_{c}=\pi / 3$

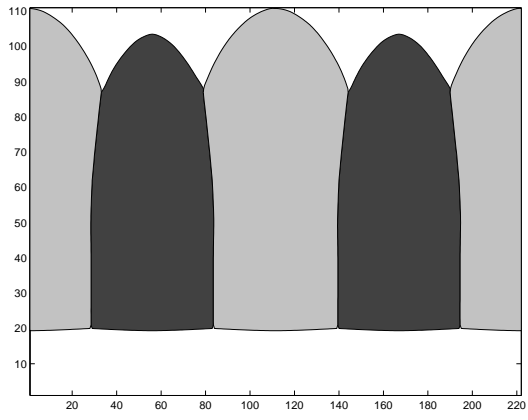

(b) $\theta_{c}=\pi / 2$

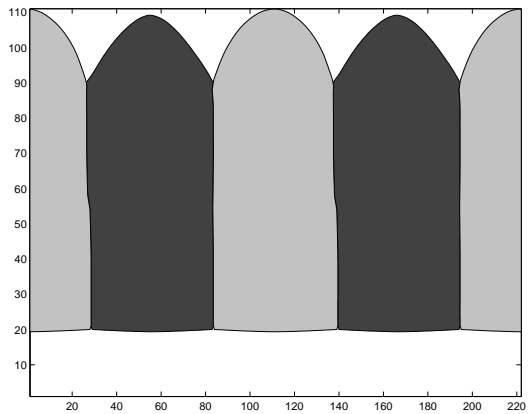

(c) $\theta_{c}=2 \pi / 3$

FIG. 10. Dark grain represents $\langle 111\rangle$, light grain represents $\langle 100\rangle, T=300 \mathrm{~K}, \alpha_{\langle 111\rangle}=\alpha_{\langle 100\rangle}=135^{\circ}$, varying $\theta_{c}$.

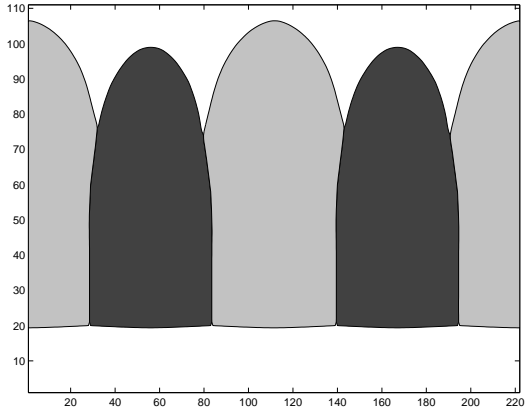

(a) $\theta_{c}=\pi / 3$

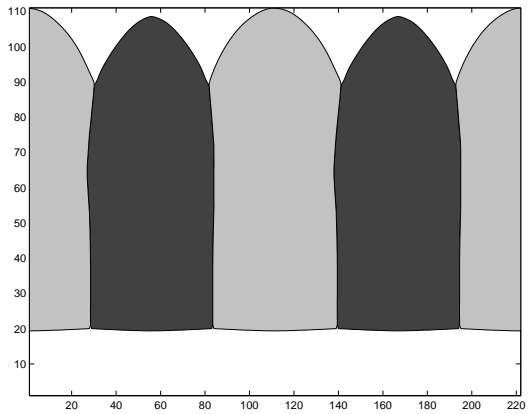

(b) $\theta_{c}=\pi / 2$

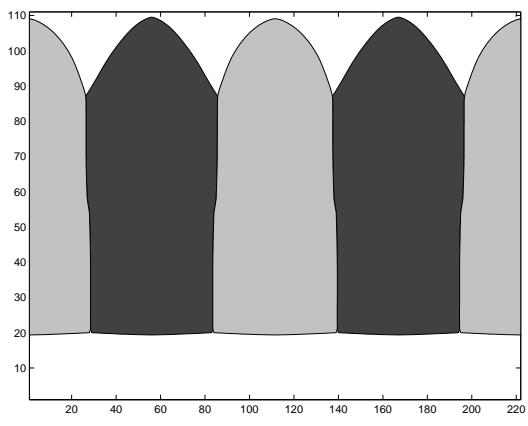

(c) $\theta_{c}=2 \pi / 3$

FIG. 11. Dark grain represents $\langle 111\rangle$, light grain represents $\langle 100\rangle, T=300 \mathrm{~K}, \alpha_{\langle 111\rangle}=100^{\circ}, \alpha_{\langle 100\rangle}=160^{\circ}$, varying $\theta_{c}$ 


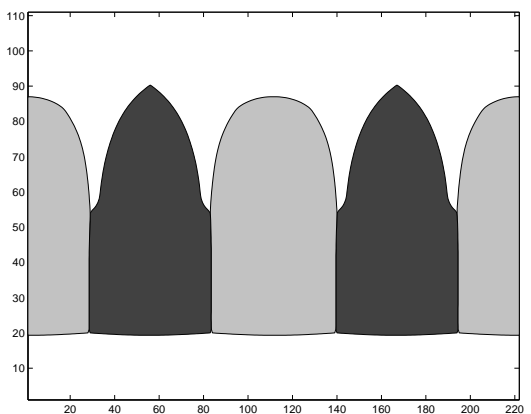

(a) $\theta_{c}=\pi / 3$

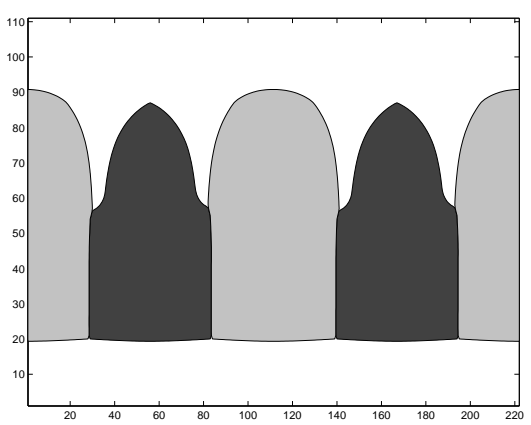

(b) $\theta_{c}=\pi / 2$

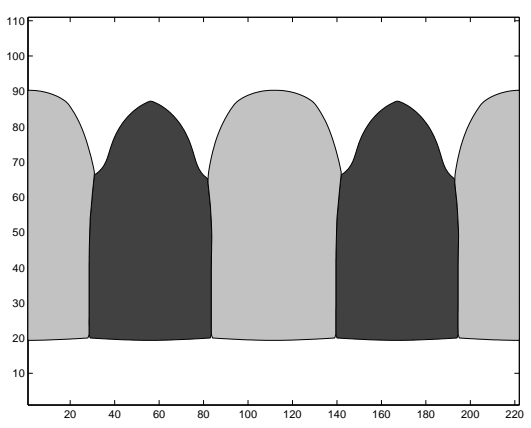

(c) $\theta_{c}=2 \pi / 3$

FIG. 12. Dark grain represents $\langle 111\rangle$, light grain represents $\langle 100\rangle, T=700 \mathrm{~K}, \alpha_{\langle 111\rangle}=\alpha_{\langle 100\rangle}=135^{\circ}$, varying $\theta_{c}$.

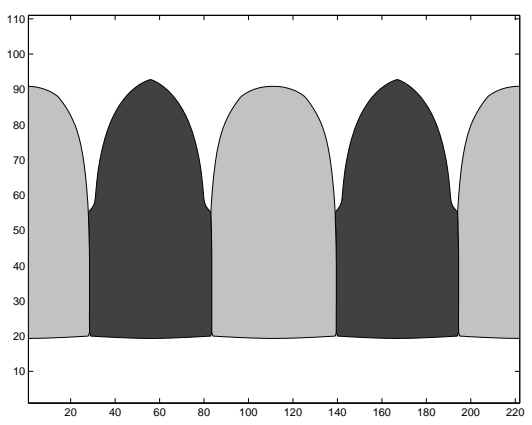

(a) $\theta_{c}=\pi / 3$

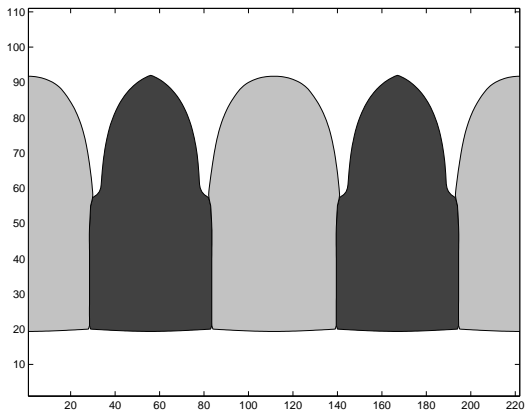

(b) $\theta_{c}=\pi / 2$

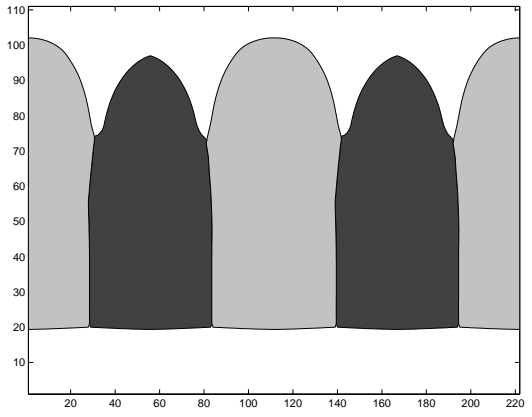

(c) $\theta_{c}=2 \pi / 3$

FIG. 13. Dark grain represents $\langle 111\rangle$, light grain represents $\langle 100\rangle, T=700 \mathrm{~K}, \alpha_{\langle 111\rangle}=100^{\circ}, \alpha_{\langle 100\rangle}=160^{\circ}$, varying $\theta_{c}$.

Next, we examine $T=700 \mathrm{~K}$, where diffusion dominates. We expect that the $\langle 100\rangle$ grain will dominate. Recall that this dominance is signified by a greater height and mass of the evolving grain.

In Figures 12 and 13, we notice that the larger contact angles once again produce the most experimentally agreeable results. Once again this result is due to the influence of material deposited at the grain boundaries. In the high temperature case, the $\langle 111\rangle$ grain displays faceting. Since anisotropic surface diffusion is the dominant component, material is pushed to the top of the grain rather than allowing the $\langle 111\rangle$ grain to take over the $\langle 100\rangle$ grain. The aforementioned physical 


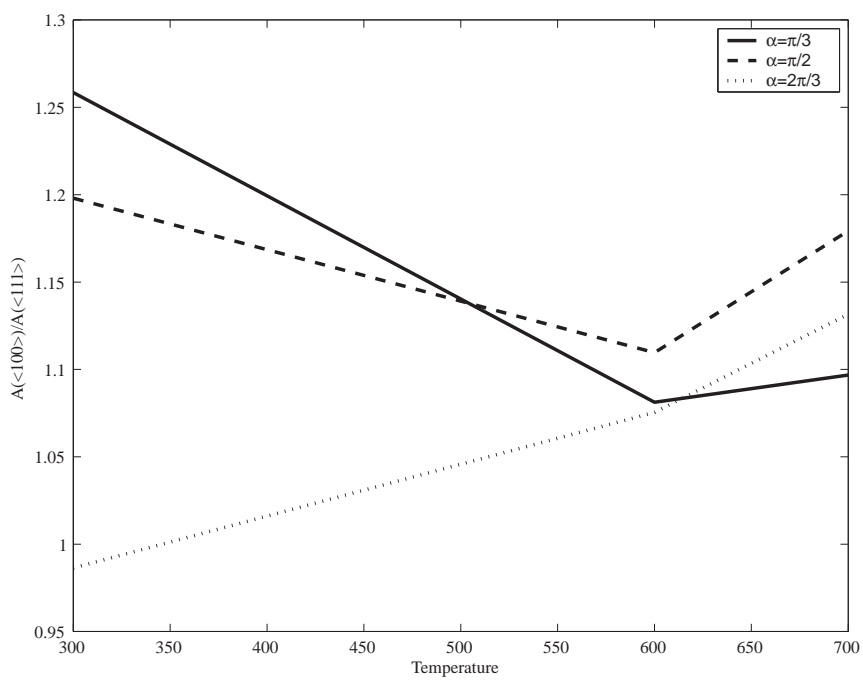

FIG. 14. Plot of ratio of areas $A(\langle 100\rangle) / A(\langle 111\rangle)$ versus temperature.

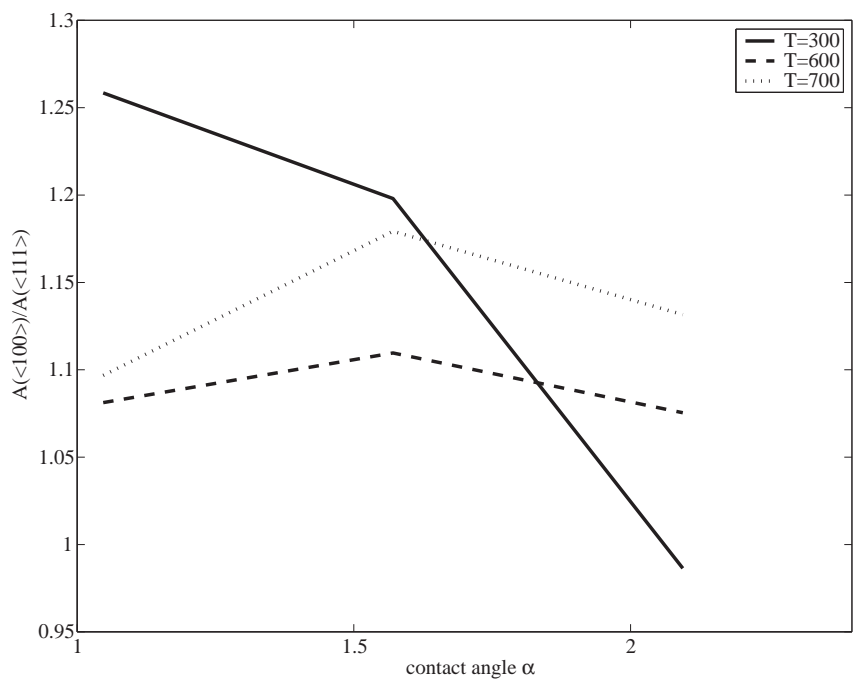

FIG. 15. Plot of ratio of areas $A(\langle 100\rangle) / A(\langle 111\rangle)$ versus contact angle.

explanation for faceting is often seen in experiments, but is usually difficult to reproduce in simulations.

The results for different contact angles and different temperatures are summarized in Figures 14 and 15 where the ratio of area for $\langle 100\rangle$ to $\langle 111\rangle$, denoted as $A(\langle 100\rangle) / A(\langle 111\rangle)$, is plotted for different temperatures and contact angles. Recall that in order for an orientation to dominate it must have greater mass and height than the other orientation. Figure 14 shows that only $\alpha=2 \pi / 3$ has a point with $A(\langle 111\rangle)>A(\langle 100\rangle)$; note that we plot data from the temperatures $T=300 \mathrm{~K}$, 
$T=600 \mathrm{~K}$, and $T=700 \mathrm{~K}$. Recall that greater area is not the only factor that displays dominance; for instance, from Figure 14 it appears that all contact angles are viable for $T=700 \mathrm{~K}$. However, examining Figure 12 shows that only $\alpha=2 \pi / 3$ has greater height along with greater area. Recall that height is extremely important due to the influence of the $1 / r^{2}$ term in the model for deposition.

Therefore, a related result is a more accurate understanding of the contact angle in grain growth. In [21] a contact angle of approximately $125^{\circ}$ is given for an iron-indium system, while a contact angle of less than $90^{\circ}$ is used for the indium particles embedded in an aluminum matrix. There is quite a disparity between these two results, so we propose that our model provides information about the impact of the contact angle on grain growth for aluminum deposited on silicon. A goal of future research is to use the present model to determine the grain evolution for different materials and numerous orientations.

Although we developed our model with surface diffusion and deposition as the two main driving forces in thin film deposition, the contact angle may be added as a third important factor. The contact angle has a large influence on the final shape of the grain and has the most influence in the deposition step. As mentioned earlier, there will be less visibility at the intersection of the grains with smaller contact angles, leading to less vertical growth. However, for larger contact angles there is more visibility near the intersection of the grains, leading to more deposition. A project of further research is to incorporate temperature into the model for the contact angle.

\section{Conclusions}

We have developed a model of multigrain thin film growth that incorporates the physically relevant parameters of orientation, surface diffusion, deposition, contact angle and temperature. In so doing, we were able to duplicate the texture competition found in experiments, while showing that the aforementioned parameters are important components for thin film growth. Having developed a robust numerical method, we are now able to extend this framework to encompass more orientations and many more grains.

We used a marker particle method to simulate anisotropic surface diffusion and the level set method to simulate deposition. Due to the nature of the experiment, in which the deposited material wets the substrate, we also accounted for a contact angle between the grains. The contact angle is critical to the behavior of the overall grain structure. In fact, for aluminum deposited on silicon, higher contact angles were more consistent with experimental observations.

The present model confirms the proposed dependence of grain growth on temperature. In the high temperature case with $T=700 \mathrm{~K}$, the $\langle 100\rangle$ orientation dominates. In the low temperature case with $T=300 \mathrm{~K}$, the $\langle 111\rangle$ orientation dominates. Even though our two-dimensional model is a simplification of a three-dimensional problem, the simulations are able to duplicate experimental results.

\section{REFERENCES}

1. Adalsteinsson, D., \& Sethian, J. A level set approach to a unified model for etching, deposition, and lithography. I: Algorithms and two-dimensional simulations. J. Comput. Phys. 120 (1995), 128-144. Zbl 0864.65086 MR 1484885

2. Adalsteinsson, D., \& Sethian, J. A level set approach to a unified model for etching, deposition, and lithography. II: Three-dimensional simulations. J. Comput. Phys. 122 (1995), 348-366. Zbl 0840.65131 MR 1484886 
3. Adalsteinsson, D., \& Sethian, J. A level set approach to a unified model for etching, deposition, and lithography. III: Redeposition, reemission, surface diffusion, and complex simulations. J. Comput. Phys. 138 (1997), 193-223. Zbl 0952.65113 MR 1484289

4. Adalsteinsson, D., \& Sethian, J. A. The fast construction of extension velocities in level set methods. J. Comput. Phys. 148 (1999), 2-22. Zbl 0919.65074 MR 1665209

5. Adam, E., Chame, A., Lancon, F., \& Villain, J. Relaxation of a grooved profile cut in a crystalline surface of high symmetry. J. Phys. I, France 7 (1997), 1455-1473.

6. Baumann, F. H., Chopp, D. L., Gilmer, G. H., Greene, J. E., Huang, H., Kodambaka, S., O'Sullivan, P., \& DE la Rubia, T. D. Multi-scale modeling of thin film deposition: Applications to Si device processing. Materials Res. Soc. Bull. 26 (2001), 182-189.

7. Bloomfield, M., Richards, D., \& Cale, T. A computational framework for modelling grainstructure evolution in three dimensions. Philos. Mag. 83 (2003), 3549-3568.

8. CAhn, J. W., \& TAYlor, J. E. Surface motion by surface diffusion. Acta Metall. Mater. 42 (1994), $1045-1063$.

9. Chang, Y., Hou, T., Merriman, B., \& Osher, S. A level set formulation of eulerian interface capturing methods for incompressible fluid flows. J. Comput. Phys. 124 (1996), 449-464. Zbl 0847.76048 MR 1383769

10. CHOPP, D. Some improvements of the fast marching method. SIAM J. Sci. Comput. 23 (2001), 230-244. Zbl 0991.65105 MR 1860913

11. Chopp, D. L. Computing minimal surfaces via level set curvature flow. J. Comput. Phys. 106 (1993), 77-91. Zbl 0786.65015 MR 1214016

12. Chоре, D. L. A level-set method for simulating island coarsening. J. Comput. Phys. 162 (2000), 104-122. Zbl 0976.82003

13. Chоpp, D. L., \& Sethian, J. A. Flow under mean curvature: Singularity formation and minimal surfaces. Tech. Rep. PAM-541, Center for Pure and Appl. Math., Univ. of California, Berkeley, 1991.

14. Chopp, D. L., \& Sethian, J. A. Motion by intrinsic Laplacian of curvature. Interfaces Free Bound. 1 (1999), 107-123. Zbl 0938.65144 MR 1865108

15. Gilmer, G. Private communication (1998).

16. Gilmer, G. Private communication (2002).

17. Gilmer, G., Huang, H., \& Roland, C. Thin film deposition: fundamentals and modeling. Comp. Mater. Sci. 12 (1998), 354-380.

18. Goetz, R., Gomprer, G., \& Lipowsky, R. Mobility and elasticity in self-assembled membranes. Phys. Rev. Lett. 82 (1999), 221-224.

19. Greene, J., Sundren, J., Hultman, L., Petrov, I., \& Bergstrom, D. Development of preferred orientation in polycrystalline tin layers grown by ultrahigh vacuum reactive magnetron sputtering. Appl. Phys. Lett. 67 (1995), 2928-2930.

20. Gyure, M. F., Ratsch, C., Merriman, B., Caflisch, R. E., Osher, S., Zinck, J. J., \& VVEDENSKy, D. D. Level-set methods for the simulation of epitaxial phenomena. Phys. Rev. E 58 (1998), R6927-R6930.

21. Howe, J. M. Interfaces in Materials: Atomic Structure, Thermodynamics and Kinetics of Solid-Vapor, Solid-Liquid and Solid-Solid Interfaces. Wiley, New York (1997).

22. Malladi, R., Sethian, J. A., \& Vemuri, B. C. Evolutionary fronts for topology-independent shape modeling and recovery. Proc. Third European Conference on Computer Vision (Stockholm, 1994), Lecture Notes in Comput. Sci. 800, Springer (1994), 3-13.

23. Mullins, W. W. Theory of thermal grooving. J. Appl. Phys. 28 (1957), 333-339.

24. Osher, S., \& Fedkiw, R. Level Set Methods and Dynamic Implicit Surfaces. Springer (2003). Zbl 1026.76001 MR 1939127 
25. Osher, S., \& SEThian, J. A. Fronts propagating with curvature-dependent speed: Algorithms based on Hamilton-Jacobi formulations. J. Comput. Phys. 79 (1988), 12-49. Zbl 0659.65132 MR 0965860

26. Penev, E., Kratzer, P., \& Scheffler, M. Effect of strain on surface diffusion in semiconductor heteroepitaxy. Phys. Rev. B 64 (2001), 085401.

27. Petrov, I., Barna, P., Hultman, L., \& Greene, J. Microstructural evolution during film growth. J. Vac. Sci. Technol. A 21 (2003), no. 5, S117-S128.

28. Russo, G., \& Smereka, P. A level-set method for the evolution of faceted crystals. SIAM J. Sci. Comput. 21 (2000), 2073-2095. Zbl 1005.82001 MR 1762031

29. Sethian, J. Level Set Methods: Evolving Interfaces in Geometry, Fluid Mechanics, Computer Vision and Material Science. Cambridge Univ. Press (1996). Zbl 0859.76004

30. Sethinan, J. A. Curvature and the evolution of fronts. Comm. Math. Phys. 101 (1985), 487-499. Zbl 0619.76087 MR 0815197

31. Sethian, J. A. Numerical algorithms for propagating interfaces: Hamilton-Jacobi equations and conservation laws. J. Differential Geom. 31 (1990), 131-161. Zbl 0691.65082 MR 1030668

32. Sethian, J. A. A marching level set method for monotonically advancing fronts. Proc. Natl. Acad. Sci. USA 93 (1996), 1591-1595. Zbl 0852.65055 MR 1374010

33. StOlARSKA, M., \& CHOPP, D. L. Modeling spiral cracking due to thermal cylcing in integrated circuits. Internat. J. Numer. Methods Engrg. 41 (2003), 2381-2410.

34. Stolarska, M., Chopp, D. L., Möes, N., \& Belytschko, T. Modelling crack growth by level sets in the extended finite element method. Internat. J. Numer. Methods Engrg. 51 (2001), 943-960. Zbl 1022.74049

35. StUmPf, R., \& SCHEFfler, M. Ab Initio calculations of energies and self-diffusion on flat and stepped surfaces of Al and their implications on crystal growth. Phys. Rev. B 53 (1996).

36. Sukumar, N., Chopp, D. L., \& Moran, B. Extended finite element method for three-dimensional fatigue crack propagation. Engrg. Fract. Mech. 70 (2003), 29-48.

37. Sussman, M., Smereka, P., \& Osher, S. A level set method for computing solutions to incompressible two-phase flow. J. Comput. Phys. 114 (1994), 146-159. Zbl 0808.76077

38. TAndia, A. Caractérisation des couches minces de $\mathrm{SiO}_{2}$ sur $\mathrm{Si}$ par simulation à l'échelle atomique. $\mathrm{PhD}$ thesis, Univ. Paul Sabatier, Toulouse (1997).

39. Tandia, A., Sarabayrousse, G., \& Martinez, A. The $\mathrm{Si} / \mathrm{SiO}_{2}$ interface morphology study by molecular simulation. Thin Solid Films 296 (1997), 122-125.

40. Vorst, G. A. L. V. D. Modelling and numerical simulation of viscous sintering. PhD thesis, Eindhoven Univ. of Technology (1994). Zbl 0789.76004

41. Wilson, S., Tracy, C., And Freeman, J. Handbook of Multilevel Metallization for Integrated Circuits. Noyes Publ. (1993).

42. Wortis, M. Chemistry and Physics of Solids Surfaces VII. Springer Ser. Surface Sci. 10, Springer, (1988).

43. Zhu, J., \& Sethian, J. A. Projection methods coupled to level set interface techniques. J. Comput. Phys. 102 (1992), 128-138. Zbl 0751.76050 